\title{
The Mbd1-Atf7ip-Setdb1 pathway contributes to the maintenance of $X$ chromosome inactivation
}

\author{
Alissa Minkovsky, Anna Sahakyan, Elyse Rankin-Gee, Giancarlo Bonora, Sanjeet Patel and Kathrin Plath*
}

\begin{abstract}
Background: $X$ chromosome inactivation $(X C l)$ is a developmental program of heterochromatin formation that initiates during early female mammalian embryonic development and is maintained through a lifetime of cell divisions in somatic cells. Despite identification of the crucial long non-coding RNA Xist and involvement of specific chromatin modifiers in the establishment and maintenance of the heterochromatin of the inactive $X$ chromosome (Xi), interference with known pathways only partially reactivates the Xi once silencing has been established. Here, we studied ATF7IP (MCAF1), a protein previously characterized to coordinate DNA methylation and histone H3K9 methylation through interactions with the methyl-DNA binding protein MBD1 and the histone H3K9 methyltransferase SETDB1, as a candidate maintenance factor of the Xi.

Results: We found that siRNA-mediated knockdown of Atf7ip in mouse embryonic fibroblasts (MEFs) induces the activation of silenced reporter genes on the $X_{i}$ in a low number of cells. Additional inhibition of two pathways known to contribute to Xi maintenance, DNA methylation and Xist RNA coating of the X chromosome, strongly increased the number of cells expressing Xi-linked genes upon Atf7ip knockdown. Despite its functional importance in Xi maintenance, ATF7IP does not accumulate on the Xi in MEFs or differentiating mouse embryonic stem cells. However, we found that depletion of two known repressive biochemical interactors of ATF7IP, MBD1 and SETDB1, but not of other unrelated H3K9 methyltransferases, also induces the activation of an Xi-linked reporter in MEFs.

Conclusions: Together, these data indicate that Atf7ip acts in a synergistic fashion with DNA methylation and Xist RNA to maintain the silent state of the Xi in somatic cells, and that Mbd1 and Setdb1, similar to Atf7ip, play a functional role in Xi silencing. We therefore propose that ATF7IP links DNA methylation on the Xi to SETDB1-mediated H3K9 trimethylation via its interaction with MBD1, and that this function is a crucial feature of the stable silencing of the Xi in female mammalian cells.
\end{abstract}

Keywords: X chromosome inactivation, Xist, H3K9 methylation, Atf7ip, Mcaf1, Mbd1, Setdb1

\section{Background}

$\mathrm{X}$ chromosome inactivation (XCI) is an X chromosomewide process of gene silencing in female mammalian cells that is a mechanism for dosage compensation of X-linked gene products between the sexes. Due to the random nature of XCI, normal adult female mammalian organisms have a roughly equal distribution of cells with an inactive $\mathrm{X}$ chromosome $(\mathrm{Xi})$ of maternal or paternal origin [1]. Random XCI is initiated in early embryonic development

\footnotetext{
* Correspondence: kplath@mednet.ucla.edu

David Geffen School of Medicine, Department of Biological Chemistry, Jonsson Comprehensive Cancer Center, Molecular Biology Institute, Eli and Edythe Broad Center of Regenerative Medicine and Stem Cell Research, University of California, Los Angeles, CA 90095, USA
}

in pluripotent epiblast cells shortly after the blastocyst implants. Once established, XCI becomes remarkably stable and is maintained for the lifetime of somatic cells, but is reversed in the cells that give rise to the germline [2-5]. Experimentally, the initiation of XCI can be recapitulated in differentiating female mouse embryonic stem cells (ESCs). Notably, despite its extreme stability in differentiated cells in vivo, the reactivation of the Xi can be achieved experimentally by induction of the pluripotent state in somatic cells either through transcription factor-induced reprogramming to induced pluripotent stem cells (iPSCs), cell fusion with ESCs, or somatic cell nuclear transfer [6-8]. 
XCI begins with the upregulation and spread of the long non-coding RNA Xist on the future Xi [2-5]. Xist, itself encoded on the $\mathrm{X}$ chromosome, is absolutely required for the initiation of XCI and its coating of the chromosome initiates a cascade of silencing events including the exclusion of RNA polymerase II from the Xist RNA-coated domain, the gain of histone H3K9me2, loss of active chromatin marks such as histone H3K4 methylation and histone acetylation, the recruitment of polycomb repressive complexes (PRC) 1 and 2 and deposition of their respective histone marks H2AK119ub1 and H3K27me3, and the gain of H4K20me1 [2-5]. After the establishment of the $\mathrm{Xi}, \mathrm{XCI}$ transitions to the maintenance phase in which the repressed state of the $\mathrm{X}$ chromosome is stabilized and permanently locked in. The maintenance phase is marked by incorporation of the histone variant macroH2A, accumulation of promoter $\mathrm{CpG}$ methylation, and transition to late replication in S-phase [9-12].

Many of the protein factors implicated in XCI were identified based on their nuclear enrichment on the $\mathrm{Xi}$ by immunofluorescence [13]. Notably, one of these Xienriched proteins is the matrix protein hnRNP U, which has been shown to be required for the Xist RNA coating of the $\mathrm{Xi}$ and the initiation of X-inactivation [14]. Yet, various chromatin modifiers implicated in XCI, including Eed (the structural subunit of PRC2), Ring1b (the E3 ligase of PRC1), G9a (a histone methyltransferase mediating H3K9 methylation), and Dnmt3a/b (the de novo DNA methyltransferases) were found to be dispensable for both initiation and maintenance of $\mathrm{X}$ chromosome silencing in mice and in in vitro cell culture systems [15-18]. Therefore, the exact role for some of these factors in random XCI still remains unclear.

Interestingly, in the course of XCI initiation, the cascade of chromatin and transcriptional changes depends on continued Xist expression and remains reversible upon experimentally induced Xist shutdown [19]. In contrast, the maintenance phase of XCI is characterized by almost complete resistance of the $\mathrm{Xi}$ to reactivation upon Xist deletion $[19,20]$. To explain this switch in Xist dependence with XCI phase, studies in differentiated female cells have described synergism between Xist RNA, DNA methylation, histone variants, and histone hypoacetylation in maintaining XCI [20-22]. For instance, assaying primary mouse embryonic fibroblasts (MEFs) harboring a GFP reporter on the $\mathrm{Xi}$ that is subject to $\mathrm{X}$-inactivation, showed reactivation in approximately $11 \%$ of cells 13 days after simultaneous deletion of Xist and Dnmt1 [20]. The contribution of Xist to silencing is considerably smaller than that of Dnmt1 as Xist deletion alone only doubled the low 'spontaneous' background rate of reactivation to $0.05 \%$, while Dnmt1 deletion alone led to approximately $5 \%$ reactivation [20]. Thus, multiple epigenetic layers act together to maintain the silenced state of the $\mathrm{Xi}$ and Xist retains some role in gene silencing in the maintenance phase that is appreciated only when other repressive modifications are inhibited. Another instance of this cooperative functional role of repressive modifications in XCI involves the histone variant macroH2A as well as Cullin3 and SPOP (both members of an E3 ligase complex that ubiquitinates macroH2A) [21,22]. Knockdown of any one of these three proteins alone does not induce activation of the Xi-linked GFP reporter, but rates of activation increase when knockdown is sensitized by a DNA demethylating agent and a histone deacetylase inhibitor [21,22].

In summary, these studies demonstrate that the $\mathrm{Xi}$ in somatic cells is relatively resistant to reactivation by interference with single known factors and that seemingly distinct silencing mechanisms act in a combinatorial fashion to 'lock-in' the heterochromatin state. Notably, among the chromatin mechanisms tested for a functional role in Xi-maintenance, DNA methylation so far appears to be the most critical pathway for maintaining the silent state of the Xi in differentiated cells [20]. However, one fundamental repressive mechanism, histone H3K9 methylation, also enriches on the Xi but its importance in XCI has not yet been clearly established, raising the question of whether this methylation mark and the enzymes involved in its deposition functionally contribute to Xi-maintenance [23-27]. Furthermore, since in many developmental systems DNA methylation and histone H3K9 methylation pathways can be dependent on one another, an interesting question is whether these two chromatin regulatory mechanisms would also be linked on the $\mathrm{Xi}$ [28].

In this study, we focused on these questions by dissecting the functional contribution of the nuclear protein ATF7IP (also known as MCAF1 for Mbd-1 Chromatin Associated Factor 1) to XCI. We chose to test the function of ATF7IP in XCI for two reasons. First, though described to function as both a transcriptional activator and repressor, ATF7IP is a particularly interesting candidate factor for a regulator of XCI because in its repressive context, it has been shown to mediate MBD1-dependent transcriptional silencing through the recruitment and catalytic activation of the histone H3K9 methyltransferase SETDB1 [29-31]. Since MBD1 (methyl-CpG DNA Binding Domain Protein 1) binds methylated DNA, this function of ATF7IP could thereby bridge the DNA methylation and histone H3K9 methylation pathways on the $\mathrm{Xi}$ [32]. Second, ATF7IP is implicated to interact with CDYL, a chromodomain-containing transcriptional co-repressor that is recruited to the $\mathrm{Xi}$ by $\mathrm{H} 3 \mathrm{~K} 9 \mathrm{me} 2$, as ATF7IP was recently identified by mass spectrometry in a CDYL pulldown in undifferentiated and differentiating mouse ESCs [27]. 
Our study identifies ATF7IP and its previously described biochemical interactors MBD1 and SETDB1 as regulators of the $\mathrm{Xi}$ in differentiated cells. We reveal this function of Atf7ip, Mbd1, and Setdb1 in a 'sensitized' approach, where we add low concentrations of 5-aza-2'-dC to Xi-reactivation assays with the rationale of destabilizing DNA methylation and therefore XCI, so that interference with candidate gene function would increase rates of Xi-reactivation in somatic cells.

\section{Results and discussion}

\section{Depletion of Atf7ip leads to Xi-reactivation in somatic} cells

Recently, the chromodomain-containing transcriptional repressor CDYL was found to associate with the $\mathrm{Xi}$, requiring the induction of differentiation and both histone H3K9me2 and H3K27me3 for its Xi-enrichment [27]. Based on its biochemical interaction with the H3K9 methyltransferase G9A, it was proposed that CDYL anchors G9A to facilitate the propagation and maintenance of the H3K9me 2 mark on the $\mathrm{Xi}$ and ensure the perpetuation of the H3K9me2 on the $\mathrm{Xi}$ during S-phase [27]. Based on the timing of $\mathrm{Xi}$ association and its independence of the silencing domain of Xist, it was suggested that CDYL-regulated processes play a role in the maintenance of XCI rather than the initiation of X-inactivation. Interestingly, Heard and colleagues also found ATF7IP (MCAF1) and its homolog ATF7IP2 (MCAF2) as one of 20 high-confidence proteins interacting with CDYL in both undifferentiated and differentiating mouse ESCs [27], leading us to test the hypothesis that ATF7IP, a protein known to link the DNA methylation and H3K9 methylation pathways in autosomal gene silencing contexts, could also function in XCI.

To begin exploring the role of ATF7IP in XCI, we first investigated the consequences of Atf7ip depletion on the maintenance of the silent state of the $\mathrm{Xi}$ in somatic cells. Knockdown of Atf7ip in MEFs reduced protein levels by approximately $75 \%$ and dramatically decreased the global nuclear protein signal as assayed by immunofluoresence (Figure 1A-C, Additional file 1: Figure S1A/B), while transcript levels were decreased by about $50 \%$ (Additional file 1: Figure S1C). The specificity of the antibody directed against ATF7IP and the specific targeting of Atf7ip by the siRNA were validated further by immunostaining and knockdown, respectively, of ectopically expressed FLAG-tagged ATF7IP. Specifically, female MEFs transduced with a retroviral vector expressing FLAG-tagged ATF7IP showed a similar nuclear distribution of the FLAG-tagged protein and the endogenous protein in immunostaining experiments (Additional file 1: Figure S1D). Furthermore, retrovirally encoded FLAGATF7IP was expressed at six-fold higher levels than endogenous Atf7ip as determined by RT-PCR, and the
Atf7ip-targeting siRNA was able to target the exogenous product and reduce its transcript levels by about 50\% (Additional file 1: Figure S1E).

To monitor the functional effect of Atf7ip knockdown on the maintenance of the silent state of the $\mathrm{Xi}$, we developed a new Xi-linked reporter that is highly sensitive for Xi-reactivation. Specifically, the firefly luciferase open reading frame under control of the constitutive CAG promoter was targeted into the Hprt locus on the single $\mathrm{X}$ chromosome in male mouse ESCs using Flp-mediated recombination, an approach similar to one described previously [33] (Additional file 2: Figure S2). Correct targeting was confirmed by southern blotting (data not shown). Subsequently, transgenic mice were generated by blastocyst injection of a targeted ESC clone. Upon germline transmission, skewing of XCI to the luciferase-bearing X chromosome was ensured by breeding male reporter mice with female mice heterozygous for an Xist knockout ( $\Delta$ Xist) allele [34]. It has been previously demonstrated that the loss of Xist on one $\mathrm{X}$ chromosome in female mice leads to inactivation of the wild-type X chromosome in $100 \%$ of the cells, thereby resulting in the Xist-deleted X chromosome remaining active (the $\mathrm{Xa}$ ) in all cells [35].

As expected, $\mathrm{Xi}^{\mathrm{CAG}-\mathrm{Luciferase}} \mathrm{Xa}^{\Delta \mathrm{Xist}} \mathrm{MEFs}$ derived from these animals (carrying the luciferase reporter on the $\mathrm{Xi}$ in all cells) did not show luciferase expression in normal culture conditions (Additional file 3: Figure S3Ai, untreated condition). By contrast, female MEFs carrying wild-type Xist on both X chromosomes and heterozygous for CAG-luciferase in the Hprt locus displayed strong luciferase expression due to random XCI (Additional file 3: Figure S3Aii). These results indicate that our new reporter is subject to developmental XCI and becomes efficiently silenced when located on the $\mathrm{Xi}$. In agreement with this finding, the luciferase reporter gene including its promoter was found to be highly methylated on the $\mathrm{Xi}$ in $\mathrm{Xi}^{\text {CAG-Luciferase }} \mathrm{Xa}^{\text {XXist }} \mathrm{MEF}$ as determined by reduced-representation bisulfite sequencing (Additional file 4: Figure S4A, B). Luciferase expression in $\mathrm{Xi}^{\mathrm{CAG}-\mathrm{Luciferase}} \mathrm{Xa}^{\Delta \mathrm{Xist}}$ MEFs increased when the DNA methylation machinery was impaired by either depleting Dnmt1 with siRNAs or treating cells with the demethylating agent 5-aza-2'-dC (Figure 1Di, Additional file 3: Figure S3Ai). Accordingly, levels of DNA methylation at the luciferase reporter decreased upon treatment with a high concentration of 5-aza-2'-dC (10 uM) (Additional file 4: Figure S4B, C). Thus, the luciferase reporter in the Hprt locus on the Xi can be activated efficiently when a known pathway of $\mathrm{Xi}$ maintenance is impaired. Therefore, we used these fibroblasts to examine the role of Atf7ip in XCI.

We found that Atf7ip knockdown alone yielded weak activation of the luciferase reporter in $\mathrm{Xi}^{\text {CAG-Luciferase }} \mathrm{Xa}^{\Delta \mathrm{Xist}}$ MEFs after three days of knockdown (at $72 \mathrm{~h}$ of treatment) (Figure 1Di). While this effect was often seen, it did 


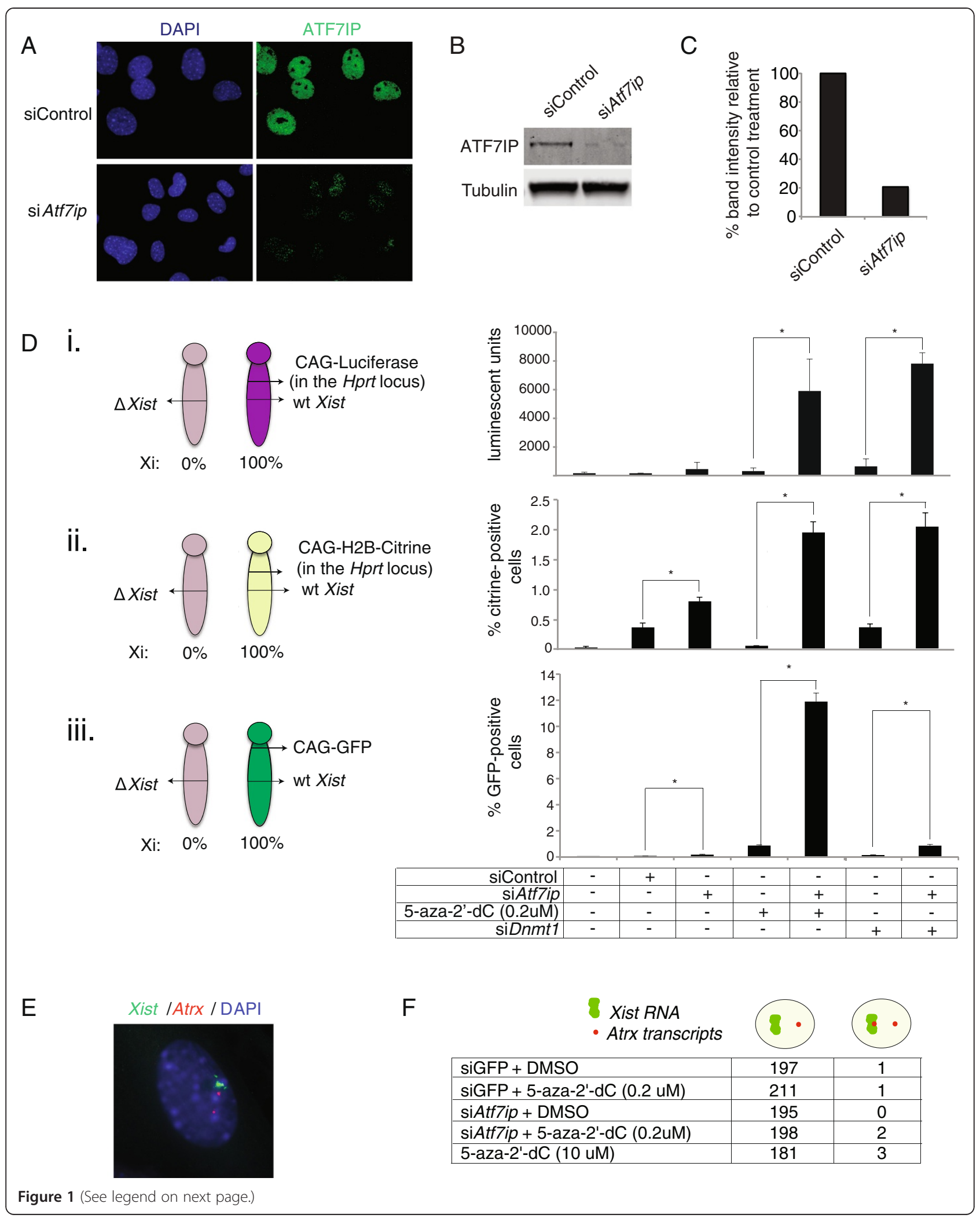


(See figure on previous page.)

Figure 1 Atf7ip is required for Xi maintenance and acts synergistically with DNA methylation. (A) Representative immunostaining images of MEFs treated with control knockdown (in this case targeting a sequence within GFP) or Atf7ip knockdown for $72 \mathrm{~h}$ and stained for ATF7IP (green). Nuclei were detected with DAPI stain. (B) Western blot of cells treated as in (A) with antibody against ATF7IP (band size approximately $220 \mathrm{kDa}$ ), with alpha-tubulin loading control. (C) Quantification of band intensity of the western blot shown in (B), normalized to alpha-tubulin

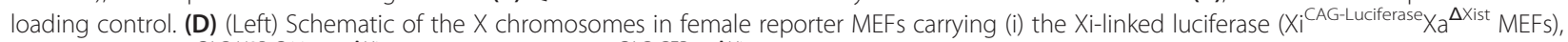
(ii) H2B-Citrine (Xi $\left.{ }^{\text {CAG-H2B-Ctirine }} X_{a}{ }^{\Delta X_{i s t}} M E F s\right)$, or (iii) GFP (Xi $\left.{ }^{C A G-G F P} X_{a}{ }^{\Delta X i s t} M E F s\right)$. (Right) Graphs summarizing the reporter assays for respective MEFs treated with control siRNA treatment (siScramble), siRNAs targeting Atf7ip and Dnmt1, and/or 5-aza-2'-dC (0.2 uM) (also referred to as low concentration 5-aza-2'-dC) as indicated, for $72 \mathrm{~h}$. Error bars indicate standard deviation of raw ALU values (for luciferase) or fluorescent cell count (for GFP and H2B-citrine) from three individual wells with the same treatment from one representative experiment. ${ }^{*}=P<0.01$ by Student's T-test. (E) RNA FISH for Xist and the X-linked gene Atrx in MEFs $72 \mathrm{~h}$ after addition of $0.2 \mathrm{um}$ (low) 5-aza-2'-dC and transfection of siRNAs targeting Atf7ip. Atrx pinpoints of transcript signal mark the site of nascent transcription. Representative image for a cell displaying expression of Atrx from the Xi, with two pinpoints for Atrx, one associated with the Xist RNA cloud. (F) Summary of counts of nuclei analyzed by RNA FISH for Atrx and Xist RNA, with the indicated FISH patterns.

not consistently pass significance tests. Since it is known that various repressive mechanisms synergistically act to maintain the silent state of the $\mathrm{Xi}$, we reasoned that combining Atf7ip knockdown with conditions of DNA demethylation may unmask an effect of Atf7ip depletion [20]. We first used the demethylating agent 5-aza-2'-dC, which inhibits DNMT1 and impedes propagation of DNA methylation through cell division [36]. On its own, 5-aza-2'-dC had a dose-dependent activity as increasing concentration induced higher rates of Xi-reactivation based on the luciferase reporter assay (Additional file 3: Figure S3Ai). In our Atf7ip knockdown experiments (Figure 1Di), we chose to add 0.2 uM 5-aza-2'-dC, since that amount of 5-aza-2'-dC induced only very little luciferase activity on its own (Additional file 3: Figure S3Ai). When combined with this low level of 5-aza-2'-dC, luciferase reporter reactivation due to Atf7ip knockdown was reproducibly enhanced up to 50 -fold (Figure $1 \mathrm{Di}$ ). To confirm that the Xi-reactivation effect of 5-aza-2'-dC treatment on Atf7ip knockdown was indeed due to changes in DNA methylation and not through potential off-target effects of 5-aza-2'-dC, we also depleted Dnmt1, the main enzyme responsible for the maintenance of DNA methylation, with siRNAs in place of 5-aza-2'-dC treatment (Additional file 3: Figure S3B). Again, we observed strong enhancement in reporter activation when Atf7ip was depleted concurrently with Dnmt1, while knockdown of either Atf7ip or Dnmt1 alone induced very low levels of luciferase activity (Figure 1Di). Addition of low levels of 5-aza-2'-dC did not significantly alter the efficacy of Atf7ip knockdown as assayed by western blot (compare Figure 1B, C to Additional file 1: Figure S1A, B). Therefore, we conclude that depletion of Atf7ip on its own has a limited effect on the $\mathrm{Xi}$, but greatly induces $\mathrm{Xi}$ reactivation when the DNA methylation maintenance pathway is impaired concomitantly. These data reveal a synergistic effect on the Xi between ATF7IP and the DNA methylation pathway.

We recapitulated these results with MEFs carrying another newly generated fluorescence reporter (histone H2B fused to citrine - H2B-citrine) in the same locus as the luciferase reporter (Hprt) (Figure 1Dii), and with MEFs carrying a previously published randomly integrated GFP transgene near the centromere on the $\mathrm{X}$ chromosome (Figure 1Diii) [20,37], allowing us to quantify the number of cells with Xi-reactivation by FACS. In both cases, depletion of only Atf7ip induced reporter activation in a low but significantly higher number of cells than control treatments. Inhibition of DNA methylation either with a low concentration 5-aza-2'-dC or by knockdown of Dnmt1 dramatically increased this effect. Taken together, the activity of Atf7ip depletion against three Xi reporters strongly supports a role for Atf7ip in the maintenance of the Xi.

We noted that reactivation in response to knockdown of Atf7ip and DNA methylation inhibition occurred in approximately $12 \%$ of GFP-reporter MEFs and in only approximately $2 \%$ of $\mathrm{H} 2 \mathrm{~B}$-citrine Xi-reporter MEFs based on flow cytometry measurements (Figure 1Dii/iii), which is potentially due to different levels of DNA methylation, other chromatin marks, and/or their integration sites. Notably, we also observed that the X-GFP reporter, when located on the Xa, was expressed at a uniformly high intensity across the cell population while, upon reactivation from the $\mathrm{Xi}$, the GFP intensity was much less uniform and ranged from low to high expression levels (Additional file 5: Figure S5A, compare blue cell population). The $\mathrm{H} 2 \mathrm{~B}$-citrine Xi-reporter displayed a similar gradient of reactivation levels upon treatment with Atf7ip knockdown and 5-aza-2'-dC (Additional file 5: Figure S5B). We believe that this gradient in fluorescence intensity under reactivation conditions is a reflection of partial reactivation of the $\mathrm{Xi}$-linked reporter transgenes that is different in each cell, likely owing to incomplete demethylation of the reporters and the interaction with persistent silencing chromatin marks.

Given the different extent of reactivation observed with our two fluorescent reporter MEF lines and the cell-tocell heterogeneity in $\mathrm{Xi}$ fluorescent reporter intensity, we next set out to determine whether Atf7ip knockdown also influenced the expression of an endogenously encoded 
X-linked gene that is subject to XCI. Specifically, we monitored the activation of Atrx by RNA fluorescence in situ hybridization (FISH) relative to the Xist RNA cloud under reactivation treatment conditions. In cells of normal ploidy, we noticed rare nuclei with two spots of nascent Atrx transcripts with one of the two spots being in proximity of the Xist RNA cloud (Figure 1E). Quantification of the FISH data suggests a rate of Atrx reactivation of $0.5 \%$ to $1.0 \%$ in response to high concentration 5-aza-2'-dC or low 5-aza-2'-dC combined with Atf7ip knockdown (Figure 1F), which contrasts the rates of Xi-reactivation of $2 \%$ to $12 \%$ that was observed with the fluorescent reporters. However, we note that the rare numbers of cells with Atrx reactivation on the Xi were not consistently observed across independent treatments and FISH counts. While the FISH data show a trend towards reactivation of an endogenous gene on the $\mathrm{Xi}$ in response to Atf7ip knockdown, we interpret the lower magnitude of reactivation as compared to the $\mathrm{Xi}$ reporter approach as stemming from poorer sensitivity of FISH compared to reporter assays. We believe that almost complete reactivation of Atrx would be required to allow the detection of a Atrx signal at the site of transcription on the Xi with our FISH methodology, but is likely not attained upon our treatments [38]. This conclusion is supported by the limited extent of reactivation of our fluorescent reporters described above (Additional file 5: Figure S5).

\section{ATF7IP does not accumulate on the $\mathbf{X i}$}

Since our results indicated that Atf7ip is crucial for the maintenance of the silent state of the $\mathrm{Xi}$ in somatic cells, particularly when DNA methylation is impaired, we next wanted to determine whether ATF7IP enriches on the $\mathrm{Xi}$ in the initiation and/or maintenance phases of XCI, employing a combined immunostaining/FISH approach. As controls, we also detected H3K9me2 and H3K27me3, which have very predictable $\mathrm{Xi}$-enrichment patterns in various phases of XCI $[27,39,40]$. To assess the initiation phase, we used previously described male ESCs with a doxycycline-inducible Xist allele [41], and combined the immunostaining for ATF7IP with FISH for Xist RNA. We found that these cells lacked an accumulation of ATF7IP on the Xist RNA-coated chromosome after $21 \mathrm{~h}$ of doxycycline treatment (Figure 2A). In agreement with previous reports, in the majority of these Xist-expressing undifferentiated ESCs, Xist RNA coating of the X chromosome was accompanied by a strong H3K27me3 enrichment, and a more infrequent and less prominent accumulation of H3K9me2 (Figure 2A, and data not shown) $[27,39,40]$. These results indicate that ATF7IP does not accumulate on the Xi during the initiation phase of XCI.

We next evaluated whether ATF7IP accumulates at later stages of XCI, by analyzing its nuclear pattern in female differentiating ESCs and female MEFs. In female ESCs induced to differentiate with retinoic acid for five days, the majority of H3K27me3/Xist RNA-demarcated $\mathrm{X}$ chromosomes was coated with H3K9me2 (as described in [27]), yet again no ATF7IP enrichment was observed (Figure 2B). Similarly, in female MEFs, where a smaller proportion of H3K27me3-demarcated inactive X chromosomes have H3K9me2 enrichment, ATF7IP displayed a nuclear staining pattern without any specific $\mathrm{Xi}$ enrichment (Figure 2C).

In summary, these data indicate that ATF7IP does not specifically accumulate on the $\mathrm{Xi}$ in any of the tested cellular systems, however this does not exclude the possibility that ATF7IP is recruited to the $\mathrm{Xi}$. It is possible that ATF7IP can fulfill its critical function in the maintenance phase of XCI as part of a dynamic interaction with the $\mathrm{Xi}$ that cannot be captured by immunostaining approaches. Alternatively, these data may suggest that ATF7IP functions on the $\mathrm{Xi}$ without being actively recruited to the Xi by Xist RNA.

\section{ATF7IP's function in $\mathrm{XCl}$ is independent of Xist}

ATF7IP was previously described to function not only as transcriptional repressor but also as transcriptional activator, as in addition to the repressive chromatin factors SETDB1 and MBD1, it was also found to interact and function with general transcription factors, RNA polymerase II, and the transcriptional activator SP1 $[42,43]$. Here, we considered the possibility that ATF7IP could directly control Xist expression, particularly because the effect of Atf7ip depletion alone (as described in Figure 1) mirrors that of Xist loss: Xist deletion alone only induces very minimal Xi-reactivation, but significantly enhances Xi-reactivation caused by DNA demethylation [20]. Therefore, it could be possible that ATF7IP functions as an activator of Xist in the context of XCI. In this scenario, depletion of Atf7ip could reduce Xist expression and coating of the chromosome by the RNA, which in turn could enhance Xi-reactivation in the presence of DNA methylation inhibitors.

We explored this idea by testing whether Atf7ip knockdown alters the Xi localization of Xist RNA, and the enrichment of the histone H3K27me3 and the chromatin regulator ASH2L, both of which are known to be recruited to the $\mathrm{Xi}$ in an Xist RNA-dependent manner $[44,45]$. Our FISH and immunostaining approaches demonstrated that Atf7ip knockdown, with or without a low dose 5-aza-2'- $\mathrm{dC}(0.2 \mathrm{uM})$, did not change the extent of $\mathrm{Xi}$ enrichment/localization of Xist RNA, H3K27me3, and Ash2l (Figure 3A, B, Additional file 6: Figure S6A, B), indicating that Atf7ip does not influence $\mathrm{XCI}$ by regulating Xist expression and the coating of the chromosome by Xist RNA, or by affecting the H3K27me3 pathway. We also compared the extent Xist RNA coating in cells that have 


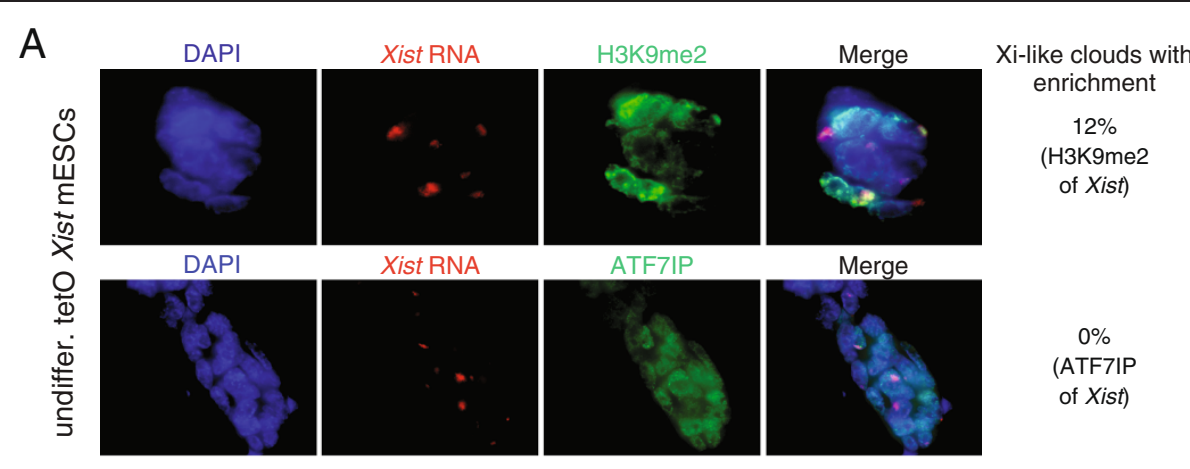

B
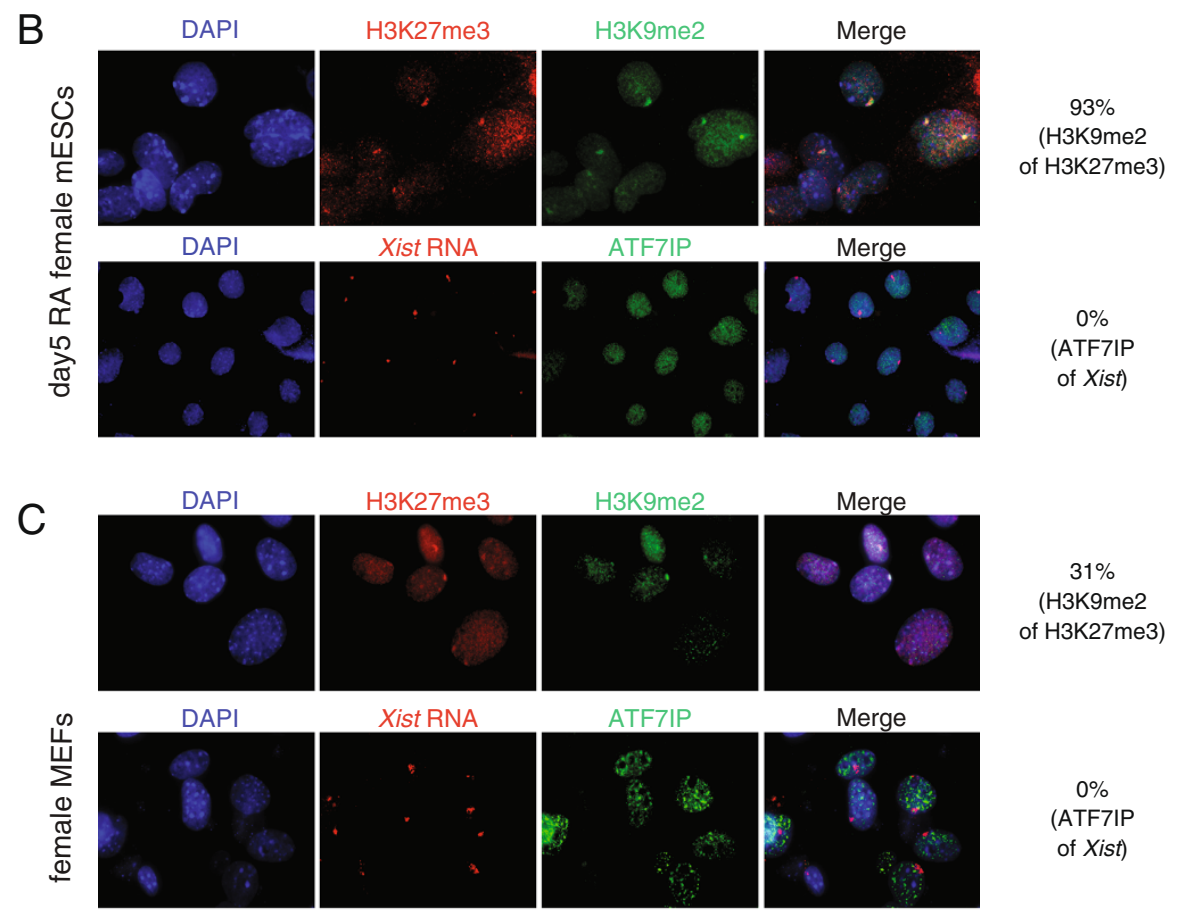

$$
\begin{gathered}
0 \% \\
\text { (ATF7IP } \\
\text { of } X i s t)
\end{gathered}
$$
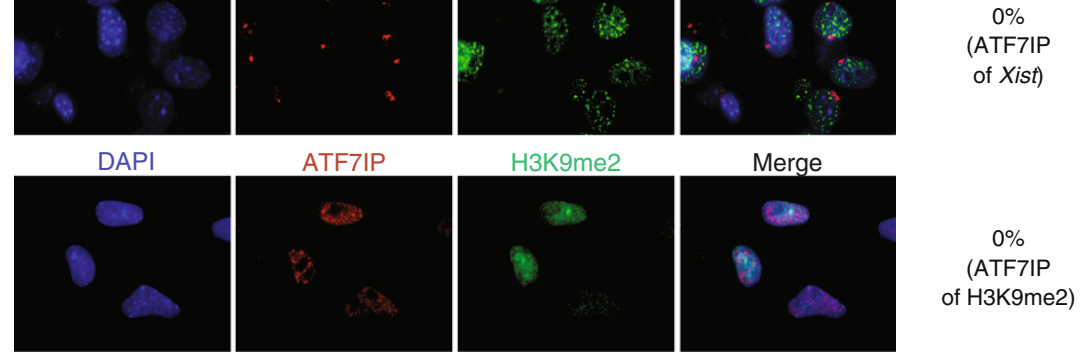

Figure 2 Nuclear localization of ATF7IP relative to Xist RNA, H3K27me3, and H3K9me2 during the initiation and maintenance phases of XCI. (A) Undifferentiated male mouse ESCs carrying a tet-inducible promoter at the endogenous Xist locus were analyzed by FISH for Xist RNA (red) and immunostaining for H3K9me2 and ATF7IP, respectively (green), $21 \mathrm{~h}$ after induction of Xist expression with doxycycline. DAPI was used to detect nuclei. Representative images are shown and the numbers on the right of the merged images indicates the percentage of cells $(n=100$ per sample) with H3K9me2 or ATF7IP enrichment within the Xist RNA-demarcated X chromosome. (B) Similar to (A), except that female mouse ESCs after 5 days of retinoic acid (RA) differentiation were analyzed by H3K27me3/H3K9me2 co-immunostaining or Xist RNA FISH in combination with ATF7IP immunostaining. (C) Similar to (A), except that female MEFs were stained for H3K9me2, H3K27me3, ATF7IP, and Xist RNA in the indicated combinations.

undergone reactivation of the Xi-linked GFP reporter versus cells that remained GFP-negative upon treatment, and did not note significant differences in Xist RNA coating (Additional file 6: Figure S6C, D). The retention of the Xist RNA cloud in cells with Xi-reactivation supports the idea that Xist RNA coating is not lost as Xi-reactivation occurs.
Since these data suggested that Atf7ip does not control Xist expression or coating, we next determined the consequences of combined knockdown of Atf7ip and deletion of Xist in MEFs on the Xi-linked H2B-citrine reporter. We hypothesized that if ATF7IP is acting in the capacity of a transcriptional repressor directly on Xi linked genes, 


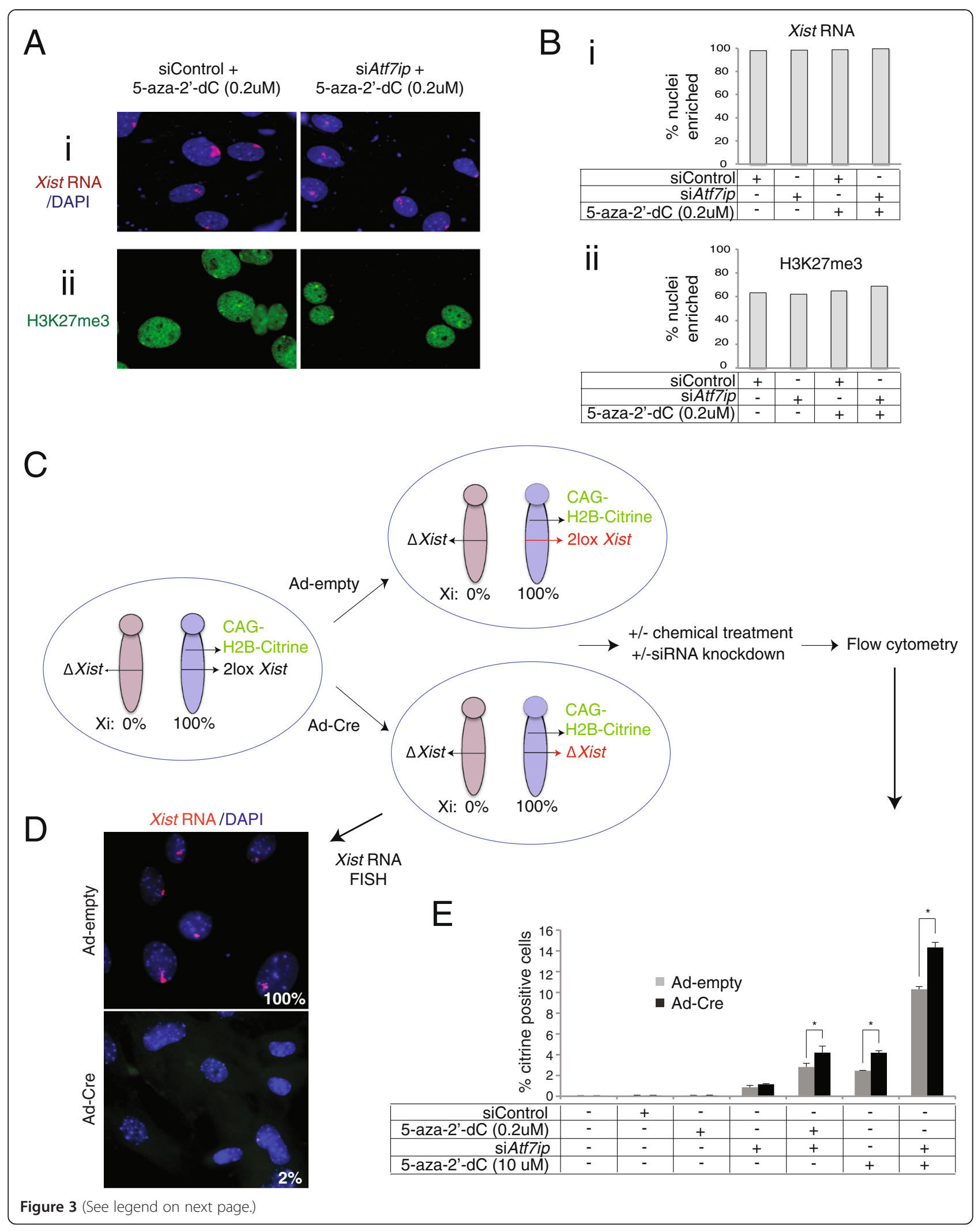


(See figure on previous page.)

Figure 3 Relationship between Atf7ip and Xist. (A) (i) Representative FISH image for Xist RNA (red) in female DAPI-stained MEFs after $72 \mathrm{~h}$ of treatment with 5-aza-2'-dC (0.2 uM) and siRNA-mediated Atf7ip knockdown or GFP (control) knockdown. (ii) As in (i), but displaying an immunostaining image for H3K27me3 under the same conditions. (B) (i) The graph depicts the percentage of DAPI-stained nuclei $(n=200$ per sample) with Xi-accumulation of Xist RNA for the conditions shown in (Ai) and additional control treatments. (ii) As in (Bi), but for the Xi-accumulation of H3K27me3. (C) Diagram of the experimental approach to test the effect of combined Xist and Atf7ip depletion on Xi reactivation. Primary female MEFs with an Xi bearing a conditional loxP-flanked allele of Xist (2lox Xist) and a CAG-driven H2B-Citrine transgene in the Hprt locus, and an Xa without a functional Xist gene were obtained by crossing mice with the respective alleles. Cells were treated with either Adenovirus(Ad)-Cre or Ad-empty (empty adenoviral vector) for $24 \mathrm{~h}$, then subjected to Atf7ip or control knockdowns and 5-aza-2'-dC treatments at various concentrations for an additional $72 \mathrm{~h}$, and analyzed by flow cytometry. (D) Representative images of Xist RNA FISH in Ad-Null and Ad-Cre treated MEFs at the time of flow cytometry. The percentage of cells with Xi-like Xist RNA accumulations is given (200 cells counted). (E) Graph representing the percentage of H2B-citrine positive cells after $72 \mathrm{~h}$ of the indicated chemical and knockdown treatments. Grey bars indicate the results for Ad-empty treated (Xist-postive) cells and black bars indicate those for Ad-Cre treated (Xist-negative) cells. As siControl we used siRNAs with a scrambled sequence in this experiment.

we would see an increase in the number of cells with $\mathrm{Xi}$ reactivation when both Atf7ip and Xist are inhibited compared to the deletion of only one of these factors. To measure the relative contributions of Xist and Atf7ip to $\mathrm{Xi-maintenance,} \mathrm{we} \mathrm{employed} \mathrm{female} \mathrm{MEFs} \mathrm{carrying} \mathrm{the}$ histone $\mathrm{H} 2 \mathrm{~B}$-Citrine reporter in the Hprt locus in cis to a conditional allele of the Xist gene on the $\mathrm{Xi}$ and assayed reactivation by FACS (Figure 3 C). Xist was deleted in half of these MEFs by infection with a Cre-recombinaseencoding adenovirus (Ad-Cre), while the other half was treated with control adenovirus (Ad-Null) (Figure 3C). Efficient loss of Xist coating in almost all of the Cre-treated cells was confirmed by FISH (Figure 3D). MEFs positive and negative for Xist, respectively, were then subjected to Atf7ip knockdown and 5-aza-2'-dC treatment for $72 \mathrm{~h}$ to assess the contribution of Xist to Xi silencing under these conditions (Figure 3C, E).

Consistent with a previous report, Xist deletion led to a roughly two-fold increase in the number of cells displaying Xi-reactivation in MEFs treated with 5-aza-2'-dC at a high concentration (10 uM) (Figure 3E) [20], confirming the role of Xist in Xi-maintenance. Reporter activation was also enhanced by Xist deletion when MEFs were depleted for Atf7ip, both with and without 5-aza-2'-dC at low concentration (0.2 um) (Figure 3E). Furthermore, with approximately $14 \%$ cells being positive for histone $\mathrm{H} 2 \mathrm{~B}$-citrine expression at $72 \mathrm{~h}$ of treatment, maximal reactivation of the $\mathrm{H} 2 \mathrm{~B}$-citrine reporter was obtained when $10 \mathrm{uM}$ (high) 5-aza-2'-dC, siAtf7ip, and loss of Xist were combined (Figure 3E). These findings further validate ATF7IP as a critical regulator of the $\mathrm{Xi}$ in somatic cells. Importantly, these observations argue against a role for ATF7IP in transcriptional activation of Xist and strongly point to an independent role in the repression of $\mathrm{Xi}$-linked genes.

\section{ATF7IP's repressive binding partners MBD1 and SETDB1 function in $\mathrm{XCl}$}

In its silencing context, ATF7IP acts as a bridging factor by binding the methyl-CpG DNA Binding Domain Protein 1 (MBD1) and the histone H3K9 trimethylase SETDB1, thereby coupling DNA methylation to histone H3K9 trimethylation and facilitating the conversion of di-methyl to tri-methyl H3K9 by SETDB1 and transcriptional repression [29-31,46]. Therefore, we hypothesized that ATF7IP could function together with MBD1 and SETDB1 on the $\mathrm{Xi}$, and next addressed whether the factors implicated in ATF7IP's autosomal gene silencing function also play a role in XCI by testing if depletion of $M b d 1$ and Setdb1 would similarly lead to Xi reactivation. Specifically, we assayed the effect of Setdb1 and Mbd1 knockdown on Xireporter activity both in the presence and absence of low concentration of 5-aza-2'- $\mathrm{dC}(0.2 \mathrm{uM})$, as we had previously observed that the effect of ATF7IP depletion was strongly enhanced by concomitant impairment of DNA methylation (Figure 1). Indeed, similar to Atf7ip depletion, knockdown of $M b d 1$ or Setdb1 activated the Xi-linked luciferase reporter more efficiently than control knockdown, both with and without low concentration of 5-aza-2'-dC (Figure 4A, B, Additional file 7: Figure S7A). Importantly, as seen before for Atf7ip, low levels of 5-aza-2'-dC dramatically increased the reactivation of the $\mathrm{Xi}$-linked luciferase reporter due to knockdown of $M b d 1$ or Setdb1 (compare Figure 4A with Figure 4B, note the different Y-scales). We also found that the simultaneous depletion of any two of these factors induced a further increase in luciferase expression from the Xi both with and without 5-aza-2'-dC, and that the depletion of all three (Atf7ip, Mbd1, and Setdb1) had the most dramatic effect (Figure 4A, B). The additive enhancement of reactivation by combinatorial knockdown is likely due to hypomorphic effect of individual siRNA knockdowns that, when superimposed, reduce the overall silencing contribution of the Mbd1-Atf7ipSetdb1 arm in the XCI pathway, or may indicate additional functions of these proteins outside of the DNA/H3K9 methylation axis. Nevertheless, these data suggest that Atf7ip acts in the maintenance of X-inactivation within the Mbd1-Atf7ip-Setdb1 pathway.

To further investigate whether the Mbd1-Atf7ip-Setdb1 pathway is a specific gene silencing mechanism that is involved in the maintenance of $\mathrm{XCI}$, and to examine whether other related silencing factors also have a role in 
A

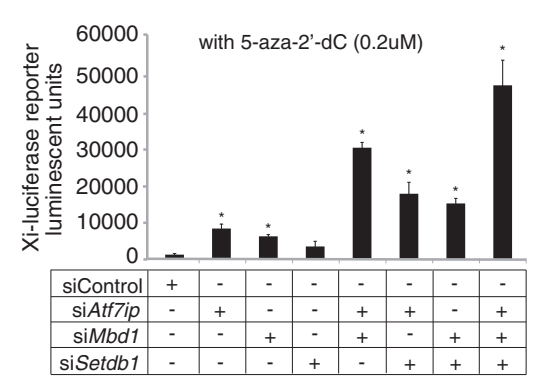

B

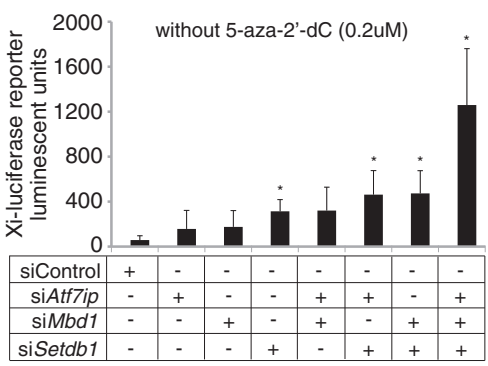

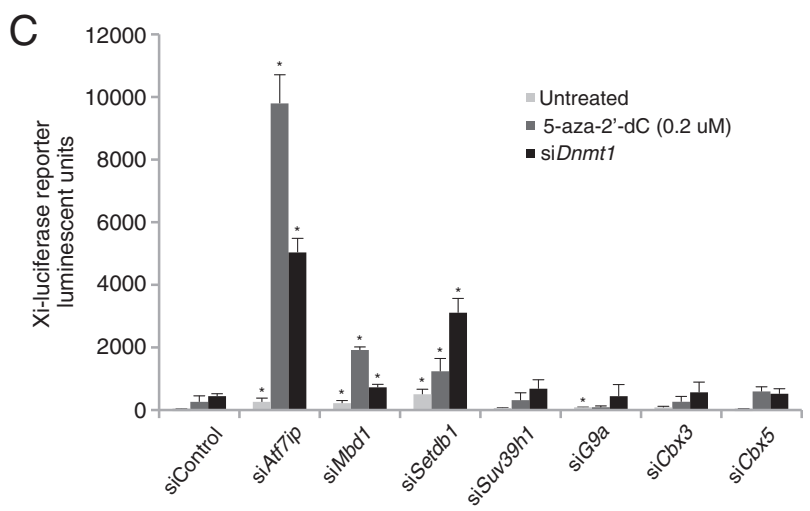

Figure 4 Knockdown of factors in the repressive Atf7ip module that link the DNA methylation and H3K9methylation pathways also

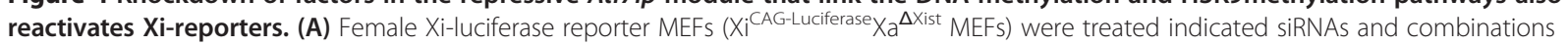
thereof in the presence of $0.2 \mathrm{uM} 5$-aza-2'-dC, and luciferase activity was measured $72 \mathrm{~h}$ later. The Graph depicts the measured raw ALU values and error bars indicate the standard deviation of values from three individual wells with the same treatment in one experiment. ${ }^{*}=P<0.05$ relative to siControl (siGFP) by Student's T-test. (B) As in (A), except that the 5-aza-2'-dC treatment was omitted. (C) As in (A), but Xi-luciferase reporter MEFs were treated with siRNAs targeting various regulators of H3K9methylation, without concomitant inhibition of DNA methylation (untreated), in the presence of $0.2 \mathrm{uM}$ 5-aza-2'-dC, or with depletion of Dnmt1 (siDnmt1), and luciferase activity was measured $72 \mathrm{~h}$ later.

$\mathrm{XCI}$, we screened a selected panel of siRNAs validated to target other mediators of transcriptional silencing including heterochromatin proteins $(C b \times 3$ and $C b \times 5)$ and other H3K9 methyltransferases (Ehmt2 (G9a) and Suv39h1) (Figure 4C) [47]. Despite efficient knockdown (Additional file 7: Figure S7B), our data do not implicate any of these additional factors in the maintenance of XCI even when tested with 5-aza-2'-dC or siDnmt1 sensitization (Figure 4C). This finding supports the conclusion that the three factors $M b d 1$, Atf7ip, and Setdb1 play a specific role in XCI.

Taken together, our results suggest that DNA methylation, the Mbd1-Atf7ip-Setdb1 pathway, and Xist RNA cooperate in maintaining the silent state of Xi-linked genes in differentiated female cells.

\section{Conclusions}

In summary, we show that depletion of Atf7ip or of its known biochemical interactors $M b d 1$ and Setdb1 leads to Xi-reactivation. We also assayed the interaction of Atf7ip with two of the known major mediators of Xi-maintenance, Xist expression and DNA methylation. Our data reveal a strongly enhanced reactivation of the $\mathrm{Xi}$ when all these mechanisms are inhibited and implicate the Mbd1-Atf7ipSetdb1 pathway, described to couple DNA methylation and histone 3 lysine 9 trimethylation, in the maintenance of $\mathrm{XCI}$ in differentiated cells.

It remains unclear from our data whether ATF7IP is necessary for XCI in vivo, as, to our knowledge, no knockout mouse model has been described yet. Atf7ip's pattern of expression is consistent with a role in XCI maintenance as in situ hybridization shows that it is ubiquitously expressed in d9.5 embryos with more specific tissue distribution at later time points [46]. Notably, ATF7IP's repressive partners MBD1 and SETDB1 have very different knockout phenotypes, namely mild spatial learning defects and autoimmune disease in adult mice lacking $M b d 1$, and early embryonic lethality at 3.5 to 5.5 days post conception for the Setdb1 deletion [48-50]. Given these different knockout phenotypes, it would be interesting to see whether Atf7ip tethers the two proteins, one binding methylated DNA and one mediating histone H3K9 trimethylation, only in specific developmental contexts, such as XCI. Notably, the effect of Atf7ip, Setdb1, or Mbd1 depletion on the Xi was more clearly unmasked when other repressive mechanisms 
were also inhibited, suggesting that the knockout animals may not reveal problems with XCI due to redundancy of silencing mechanisms acting on the Xi.

The identification of Atf7ip as a factor involved in XCI maintenance suggests that the downstream effect of its binding partner Setdb1, namely enzymatic conversion of H3K9me2 to H3K9me3, may also be required on the Xi. H3K9 methylation has previously been reported to play a role in XCI on the basis of enrichment on the Xi detected with a pan-methyl H3K9 antibody in mouse and human cells, though it is unclear if the antibody used in these studies had cross-reactivity to H3K27me3 [23-25]. Other studies comparing H3K27me3 and H3K9me2 by ChIP and by immunostaining established that both marks are increased on the $\mathrm{Xi}$ in MEFs relative to the active $X[26,27]$ (Figure 2). In human cells, the Xi has an H3K9me3 domain distinct from the Xist and H3K27me3enriching territory of the $\mathrm{Xi}$ [51]. Interaction of these domains is proposed to occur through the $\mathrm{HBiX}$ protein bridging HP1, a protein binding HK9me3, and SMCHD1, a protein required for methylation of $\mathrm{CpG}$ islands on the $\mathrm{Xi}$ and recruited by Xist to the H3K27me3 domains [52]. This bridging of H3K27me3 and H3K9me3 domains is thought to be necessary for Xi compaction, however, depletion of HBiX or SMCHD1 by siRNA did not lead to upregulation of Xi-linked genes [52], leaving an open question as to whether H3K9me3 has silencing function in XCI and whether that function is dependent on HP1 and HBiX binding.

It remains unclear whether knockdown of Setdb1, though a known histone H3K9 trimethyltransferase, would deplete H3K9me3 on the Xi, since the Setdb1 knockout has not been shown to lead to global alterations in H3K9me3 levels nor DNA methylation in early embryonic mouse cells when assayed by immunostaining and bisulfite sequencing of IAP elements, respectively [48]. In addition, the H3K9 methyl reader heterochromatin proteins $\mathrm{Cbx} 1, \mathrm{Cbx} 3$, and/or Cbx 5 are thought to mediate transcriptional repression downstream to H3K9 methylation, yet, in our study, knockdown of $C b x 3$ or $C b x 5$ individually had no effect on the reactivation of the Xi in somatic cells (Figure 4C). Furthermore, we did not see strong Xi enrichment of Atf7ip or H3K9me3 by immunostaining (Figure 2, Additional file 8: Figure S8). However, we observed that in a fraction of cells a subdomain of the H3K27me3-demarcated Xi co-localizes with a punctum of H3K9me3 enrichment (Additional file 8: Figure S8). ChIP-seq studies of H3K9me3 and the analysis of DNA methylation patterns with and without knockdown of Atf7ip, Setdb1, and Mbd1 will be necessary to refine the role of the Atf7ip/Setdb1/Mbd1 pathway on the $\mathrm{Xi}$ and to determine whether genes on the $\mathrm{Xi}$ have different propensities for regulation through this pathway. To explain the combinatorial effect of DNA methylation with interference of the Mbd1-Atf7ip-Setdb1 pathway, we hypothesize that some DNA methylation loss is necessary to 'unlock' the stably silenced chromatin. However, the relationship between H3K9me3 and DNA methylation may be reciprocal and $\mathrm{H} 3 \mathrm{~K} 9 \mathrm{me} 3$ could also recruit the DNA methylation machinery [53].

Finally, ATF7IP has also been shown to interact with CDYL, a protein implicated in the maintenance of H3K9me2 on the $\mathrm{Xi}$, which requires H3K27me3 and H3K9me2 for its Xi accumulation on the Xi [27]. Therefore, Atf7ip may contribute to an extensive regulatory network that links various repressive chromatin pathways to contribute to the maintenance of the $\mathrm{Xi}$.

\section{Methods}

\section{Cell culture methods}

Mouse ESCs were grown on irradiated DR4 MEFs in standard media (DMEM supplemented with 15\% FBS, nonessential amino acids, L-glutamine, penicillin-streptomycin, $\beta$-mercaptoethanol, and $1000 \mathrm{U} / \mathrm{mL} \mathrm{LIF})$. For induction of differentiation, cells were feeder-depleted for $45 \mathrm{~min}$ and subsequently plated at a density of $5.0 \times$ $10^{4}$ cells/ 6-well in MEF media (same as ESC media except that 10\% FBS were used and LIF was omitted). One day later, MEF media was supplemented with $1 \mu \mathrm{M}$ all-trans retinoic acid (Sigma) and refreshed every 2 days, for a total of 5 days. Male ESCs carrying the tet-inducible promoter in front of the endogenous Xist allele were induced to differentiate with $1 \mu \mathrm{g} / \mathrm{mL}$ doxycycline in standard ESC media for $21 \mathrm{~h}$ [41]. MEFs were cultured in MEF media and derived at embryonic day 14.5 from timed matings of mice with the appropriate genotypes (see below).

\section{Generation of X-linked reporter MEFs}

Hprt-CAG-Luciferase and Hprt-CAG-H2B-citrine reporters were engineered in male V6.5 ESCs. These ESCs were modified by two-step targeting as described in Additional file 2: Figure S2, closely following a strategy originally described for the Col1A locus in [33]. First, an FRT-flanked neomycin resistance cassette and a hygromycin resistance gene that has an FRT site embedded in its 5'coding region but lacks a promoter and an ATG initiation codon, were placed into Hprt locus by homologous recombination, deleting 1,313 bp of Hprt sequence between homology arms including the third exon of Hprt. To place the 'FRT-neo-FRT-hygro' cassette into the Hprt locus, the ColA1 homology arms of the CollA-FRT-hygro-pA 'homing' vector described in [33] were replaced with homology regions targeting the Hprt locus. The Hprt-FRT-hygro-pA 'homing' vector was introduced into V6.5 ESCs by electroporation followed by selection with $350 \mathrm{ug} / \mathrm{mL}$ G418. DNA from picked clones was analyzed for proper targeting by southern blotting using a BglII digest as outlined in Additional file 2: Figure S2. Second, using FLPe-mediated recombination, the CAG-luciferase or 
CAG-H2B-citrine transgene was introduced into one ESC clone carrying the Hprt-FRT hygro-pA 'homing' site, specifically into the FRT site in front of the hygromycin open reading frame, using the plasmids pBS32CAG-luciferase or pBS32-CAG-H2B-citrine as shown in Additional file 2: Figure S2. Co-electroporation of these plasmids with a plasmid expressing Flpe recombinase was followed by hygromycin treatment selecting for ESC clones that have the pgk promoter driving the hygromycin resistance gene due to integration of the entire pBS32 plasmid sequence. DNA from selected clones was digested with BglII and screened by southern blotting using 3' external probe for correct FLP recombination (Additional file 2: Figure S2, data not shown). The pBS32 vector was made by exchanging the tetracyclineresponsive operating binding sequence in the pgkATGfrt vector described in [33] with the constitutive CAG promoter. Luciferase was introduced into pBS32 by Gateway ${ }^{\circ}$ cloning (Life Technologies). To make pBS32 vector compatible for Gateway cloning (pBS32-GW), a Gateway cassette with attR sites and a ccdB gene was flanked by SgrAI restriction sites and ligated into a unique EcoRI site on pBS32. One-step BP and LR cloning was performed with pDonr221 entry vector, attB-primer amplified firefly luciferase from pGL3 vector (Promega) and pBS32-GW. The pBS32-CAG$\mathrm{H} 2 \mathrm{~B}$-citrine was constructed analogously using a H2Bcitrine template kindly provided by Michael Elowitz (Caltech). Luciferase and $\mathrm{H} 2 \mathrm{~B}$-citrine reporter ESCs were microinjected by the UCLA transgenic core into C57BL/6 blastocysts to produce chimeric mice following standard procedures. High agouti coat color male chimeras were bred with C57BL/6 females for germline transmission. For skewing of XCI to the luciferase-bearing X chromosome, $\mathrm{X}$-linked reporter male mice were bred with female mice heterozygous for an Xist knockout ( $\Delta X i s t)$ allele [34]. For the experiments requiring simultaneous deletion of Xist and reporter quantification, 2lox Xist [54] and Hprt-H2BCitrine alleles were bred until a spontaneous recombination event brought the alleles in cis. The recombinant males were subsequently bred to $\Delta X i s t$ females to skew $\mathrm{X}$ inactivation to the reporter-bearing $\mathrm{X}$ chromosome in the embryos. All animal experiments were in accordance with the legislation of the UCLA Animal Research Committee.

\section{Knockdown, overexpression, and chemical treatments of MEFs}

For Xi-reactivation assays, MEFs at passage 1 or 2 postderivation were seeded at a density of 60,000 cells per 12 -well well and chemicals in MEF media were added and incubated for $72 \mathrm{~h}$ before further analysis (by FISH, immunostaining, or reporter assays). 5-aza-2'-dC (Sigma A3656) was dissolved in DMSO and added at indicated concentrations. Across all conditions in a given experiment, final DMSO concentration (always below $0.1 \%$ ) and final volumes of media were kept constant across samples. Knockdown with siRNA was performed by reverse transfection at $25 \mathrm{nM}$ final concentration of siRNA. Briefly, a cell suspension was added to a pre-incubated mixture of Lipofectamine RNAimax, $100 \mathrm{uL}$ of reduced serum Opti-MEM media, and siRNA. siRNAs used in this study included Atf7ip (Ambion, AM16706), Dnmt1 (Ambion, AM161526), Mbd1 (Dharmacon, MU-05682901), Setdb1 (Dharmacon, D-040815-04), Suv39h1 (Dharmacon MU-046141-10), Ehmt2 (Dharmacon, MU-053728-03), Cbx3 (Dharmacon MU-044218-02), Cbx5 (Dharmacon MU-040799-02), Luciferase (Dharmacon, D-001210-02), Scramble (Ambion, AM4636), and GFP (Dharmacon, P002048-01). For experiments involving multiple knockdowns, control siRNA (against either Scramble, GFP, or Luciferase depending upon experiment as indicated in figure legends) was added to equalize the final siRNA concentration across all conditions. For overexpression of Flag-tagged Atf7ip, the Atf7ip cDNA was introduced into the pMX retroviral vector by In-Fusion ${ }^{\circledR}$ cloning (Clontech) of the Atf7ip cDNA and incorporation of the FLAG tag by PCR at the N-terminal end. The pMX retrovirus was generated in transfected platE cells, and MEFs were transduced as previously described [7].

\section{Luciferase assay}

Chemical and/or siRNA treatments of MEFs were performed in triplicate 12-well wells for $72 \mathrm{~h}$ and cells were lysed with $200 \mathrm{uL}$ passive lysis buffer (PLB, Promega) for $20 \mathrm{~min}$ at room temperature on an orbital shaker. Lysates were cleared by $30 \mathrm{~s}$ of centrifugation at $12,000 \times g$ and $20 \mathrm{uL}$ were assayed for luciferase activity with $50 \mathrm{uL}$ of LARI reagent (Promega) on a GloMax microplate luminometer (Promega).

\section{RT-qPCR analysis}

Cells were harvested from a 6-well format in Trizol (Invitrogen) and RNA purification was performed with the RNeasy kit (Qiagen) according to manufacturer's instructions with on-column DNAse treatment (Qiagen). cDNA was prepared using SuperScript III (Invitrogen) with random hexamers and RT-qPCR was performed using a Stratagene Mx3000 thermocycler with primers listed in Additional file 9: Table S1. Results were normalized to Gapdh by the $\Delta \mathrm{Ct}$ method.

\section{Western blot analysis}

$\mathrm{Xi}$-luciferase MEFs were treated as described above and one well was used to confirm luciferase reactivation. Cells in the other well were lysed in RIPA buffer and sonicated (30 s on, $30 \mathrm{~s}$ off, cycled for $5 \mathrm{~min}$ ). Equal volume from each sonicated lysate was loaded onto a $4 \%$ to 12\% 10-well 1.5 mm Bis-Tris gel (NuPAGE). Proteins were transferred onto a nitrocellulose membrane overnight at 
$10 \mathrm{~V}$ in $4^{\circ} \mathrm{C}$. Membranes were incubated overnight at $4^{\circ} \mathrm{C}$ with 1:2,500 dilution of polyclonal rabbit-anti-Atf7ip antibody (Nakao lab). Monoclonal mouse anti-alpha-Tubulin (Calbiochem CP06) was used as loading control at 1:1,000 dilution overnight at $4^{\circ} \mathrm{C}$. Quantification was performed on the Odyssey scanner (Li-Cor) with Image Studio software (Li-Cor) and band intensity was normalized to alphaTubulin loading control.

\section{Immunofluorescence, FISH, and chromosome paint analysis}

Cells were plated on glass coverslips, washed once with PBS, and fixed for $10 \mathrm{~min}$ in 4\% paraformaldehyde [7]. Immunostaining with antibodies against ATF7IP (Abcam 84497), H3K27me3 (Active Motif 39155), H3K9me2 (Cosmo Bio MCA-MABI0007-100-EX), H3K9me3 (Active Motif 39161, AbCam ab8898), Ash2L (Bethyl a300-107a), FLAG M2 (Sigma F3165), GFP (Nacalai USA Inc., 04404-84), and FISH with DNA probes against Xist and Atrx and IF/FISH combinations thereof were performed as previously reported and mounted with Prolong Gold reagent with DAPI [38]. BAC templates for Atrx FISH probes were obtained from the BACPAC resource (Atrx RP23-265D6).

\section{Flow cytometry}

Cells were trypsinized, washed in PBS, loaded through cell strainer caps (BD Biosciences) and analyzed on a FACSDiva machine (BD Biosciences) with FlowJo software (Tree Star, Inc.).

\section{Additional files}

Additional file 1: Figure S1. Validation of the Atf7ip knockdown approach. This figure includes western blot and immunostaining images to support knockdown of ATF7IP protein and transcript levels. (A) Western blot of cells treated as in Figure 1A, except in the presence of 5-aza-2'-dC (0.2 uM) with an antibody against ATF 7IP (band size approximately $220 \mathrm{kDa}$ ), with alpha-tubulin loading control. (B) Quantification of band intensity from the western blot shown in (A) normalized to alpha-tubulin loading control. (C) Female MEFs were treated with siRNAs targeting Atf7ip and Luciferase (siControl), and Atf7ip transcript levels were determined by RT-qPCR. The data were normalized to the control treatment and to Gapdh expression. Error bars indicate one standard deviation from three independent experiments. (D) Representative immunostaining image for ATF7IP (green) and FLAG (red) on female MEFs infected with a retrovirus encoding FLAG-tagged Atf7ip (pMX-Atf7ip), $72 \mathrm{~h}$ after infection. DAPI marks the nuclei. Note, in the image only the nucleus on the left expresses FLAG-Atf7ip and is therefore detected with the FLAG-antibody. (E) MEFs were infected with the pMX-Atf7ip retrovirus as indicated and, $72 \mathrm{~h}$ later, treated with siRNAs targeting Atf7ip and siControl (targeting luciferase or GFP), respectively, for another $72 \mathrm{~h}$. Subsequently, Atf7ip transcript levels were determined by RT-qPCR. The data were normalized to the siControl treatment and to Gapdh expression. Error bars indicate one standard deviation from three independent experiments. ${ }^{*}=P<0.01$ by Student's T-test.

Additional file 2: Figure S2. Construction of the $X$-linked luciferase reporter. This figure includes a diagram of targeting strategy to generate $X$-linked reporter transgenic mice. Targeting strategy for the generation of X-linked reporter ESCs. Top: Schematic representation of the Hprt locus on the $\mathrm{X}$ chromosome, with exons shown as gray boxes, and locations of the homology arms used to recombine the FRT-Hygro-pA 'homing cassette' in mouse ESCs. Additionally, the location of the $3^{\prime}$ probe and Bglll restriction enzyme digest strategy used in Southern bloting to confirm targeting, are indicated. Note that this targeting strategy deletes exon 3 of Hprt. Middle: The luciferase CDNA was cloned into the pBS32 vector. Bottom: Co-electroporation of the pBS32-CAG-Luciferase vector and a FLPe expression vector into FRT-Hygro-pA 'homing cassette' - bearing mouse ESCs, and subsequent hygromycin selection ensures the survival of ESC clones with recombination of the FRT sites, leading to loss of the PGKneopA cassette and insertion of luciferase gene (as described in [33]).

Additional file 3: Figure S3. The X-luciferase reporter is subject to $\mathrm{XCl}$ and sensitive to loss of DNA methylation. This figure includes a luciferase assay from the resulting transgenic MEFs to demonstrate that the X-linked luciferase reporter is silenced when located on the $X i$ and reactivates in response to interference with DNA methylation. (A) Top: Schematic of the $X$ chromosomes in female MEFs carrying (i) the heterozygous luciferase reporter transgene (as described in (A)) on the $\mathrm{Xi}$ in $100 \%$ of the cells

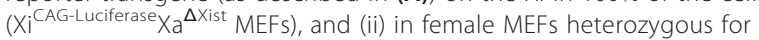
the luciferase reporter transgene without interference of Xist function, so that the luciferase reporter has a $50 \%$ chance of being silenced as part of the Xi. Bottom: Graph summarizing the luciferase values for MEFs displayed above (separated by dashed line) treated with 5-aza-2'-dC at the indicated concentrations (or DMSO vehicle) for $72 \mathrm{~h}$. Error bars indicate standard deviation of raw ALU values from three individual wells with the same treatment condition from one representative experiment. Note, luciferase values are close to background when the reporter is on the $X i$ in all cells in the population (left, untreated), but increase when DNA methylation is impaired. In cells with random XCI (right, untreated), high luciferase signal can be detected in the untreated condition since approximately half the cells express the reporter from the Xa. (B) MEFs were treated with siRNAs targeting Dnmt1 and siControl (siScramble) as described in Figure 1C, D, and Dnmt1 transcript levels were determined by RT-qPCR. The data were normalized to the siControl treatment and to Gapdh expression. Error bars indicate one standard deviation from triplicate RT-qPCR measurements in one representative experiment.

Additional file 4: Figure S4. DNA methlyation levels at the luciferase reporter gene in $X_{i}{ }^{\text {CAG-Luciferase }} X_{a}{ }^{\Delta \text { Xist }} M E F S$. This figure summarizes reduced representation bisulfite sequencing data for the reporter locus before and after treatment with 5-aza-2'-dC. (A) IGV browser view of reduced representation bisulfite sequencing data within the approximately $3 \mathrm{kB}$ reporter transgene (refer to Additional file 2: Figure S2) from two independent batches of $X_{i}{ }^{\text {CAG-Luciferase }} \mathrm{X}_{a}{ }^{\Delta X_{i s t}} \mathrm{MEFs}$. The height of the blue bars represents $\%$ methylation at the individual $\mathrm{CpG}$. Sites covered by RRBS are indicated. (B) Histograms of single CpG RRBS DNA methylation values across the luciferase reporter insert on the $X_{i}$ in untreated $X_{i}^{\text {CAG-Luciferase }} X_{a}{ }^{\Delta X_{\text {ist }}}$ MEFs. The number of CpGs covered by RRBS is given. (C) As in (B), except that the methylation values for $\mathrm{Xi}^{\text {CAG-Luciferase }} \mathrm{Xa}{ }^{\Delta \mathrm{X} \text { ist }} \mathrm{MEFs}$ treated with 5-aza-2'-dC (10 uM) are shown, indicating a drop in overall methylation compared to (B).

Additional file 5: Figure S5. Effect of Atf7ip depletion on the expression of the Xi-GFP and Xi-linked H2B citrine reporter. This figure parallels Figure 1, and shows the actual fluorescent intensities of the Xi-linked fluorescent reporters upon various treatments. (A) Top: (Left) Schematic of the $X$ chromosomes in female reporter MEFs heterozygous for the CAG-GFP reporter. Due to random XCl, the GFP is on the $X_{i}$ in approximately half the population. (Right) When in conjunction with the Xist knockout allele, the CAG-GFP reporter is on the Xi in 100\% of the cells $\left(X_{i}{ }^{\text {CAG-H2BCitrine }} X_{a}{ }^{\Delta X_{i s t}}\right)$ due to skewing with Xist deletion. Bottom: GFP fluorescence was quantified by flow cytometry in the MEFs depicted above, treated with the indicated conditions for $72 \mathrm{~h}$, and the FACS results are displayed. (B) (i) Schematic of the X chromosomes in female reporter MEFs carrying a heterozygous H2B-Citrine reporter (within the Hprt locus) on the $X_{i}$ in all cells of the population ( $X_{i}{ }^{C A G-H 2 B C i t r i n e} X_{a}{ }^{\Delta X}$ ist $M E F s$ ). (ii) Citrine fluorescence was quantified by flow cytometry in these MEFs treated with the indicated conditions for $72 \mathrm{~h}$. Note siControl knockdown produced significant background citrine fluorescence signal compared to the untreated sample. (iii, iv) FACS plots for data summarized in (ii) with initial cell gating (iii) and citrine-positive cell gating shown (iv).

Additional file 6: Figure S6. Atf7ip does not control the Xistdependent accumulation of the chromatin regulator $\mathrm{ASH} 2 \mathrm{~L}$ on the Xi. 
This figure supports Figure 2 with FISH and immunostaining to demonstrate that Atf7ip knockdown, with or without a low dose 5-aza-2'-dC $(0.2 \mathrm{uM})$, does not change the extent of Xi enrichment/localization of Ash21. It also shows that rates of Xist RNA coating of the Xi are not affected in cells that display the reactivation of the Xi-linked-GFP reporter. (A) Immunostaining for ASH2L (red) on MEFs $72 \mathrm{~h}$ after transfection of siRNAs targeting Atf7ip or control knockdown (targeting GFP), respectively, and treatment with 5-aza-2'-dC (0.2 uM) similar to the experiment described in Figure 2A. Representative images are shown and DAPI was used to demarcate the nucleus. (B) Graph summarizes the proportion of DAPI-stained nuclei ( $n=200$ per sample) with and Xi-like accumulation of ASH2L for the experiment described in (A) and additional conditions. (C) $X_{1}^{C A G-H 2 B C i t r i n e} X_{a}{ }^{\Delta X_{i s t}} M E F s$ were treated for $72 \mathrm{~h}$ with Atf7ip siRNA and 5-aza-2'-dC (0.2 um) or with 5-aza-2'-dC $10.0 \mathrm{uM}$ alone. Given is a representative IF/FISH image for Xist (red) and GFP (green) that depicts a cell with GFP reactivation that contains an Xist RNA coated chromosome. (D) Quantification of Xist RNA coating in GFP + and GFP-negative cells from the experiment described in (C), indicating the Xist RNA coating is maintained in cells displaying reactivation of the Xi-linked GFP.

Additional file 7: Figure S7. Confirmation of knockdown of Atf7ip-related factors. This figure supports Figure 4 to show that extent of knockdown of various transcripts by RT-PCR in the same experiments. (A) MEFs were treated with siRNAs targeting the indicated genes or siControl (siGFP) as described in Figure 4 and respective transcript levels were determined by RT-qPCR (that is, $40 \%$ of Atf7ip transcription remaining after siAft7ip treatment, $40 \%$ of Mbd1 transcript remaining after siMbdl treatment, and so on). The data were normalized to the siGFP (control) treatment and to Gapdh expression. Error bars indicate one standard deviation from triplicate RT-qPCR measurements in one representative experiment. (B) As in (A), but for the experiment shown in Figure $4 \mathrm{C}$ in the untreated condition (without 5-aza-2'-dC or siDnmt1).

Additional file 8: Figure S8. $\mathrm{H} 3 \mathrm{~K} 9 \mathrm{me} 3$ localization relative to the H3K27me3 Xi-domain. This figure contains immunostaining images for H3K9me3 relative to H3K27me3 to identify the Xi. Representative immunostaining images of female MEFs for H3K9me3 (green, with two different antibodies termed Active Motif and AbCam) and H3K27me3 (red) with zoomed-in views of a single nucleus. DAPI was used to demarcate the nuclei.

Additional file 9: Table S1. Primers for RT-PCR. Primer sequences used for RT-PCR analysis.

\section{Competing interests}

The authors declare that they have no competing interests.

\section{Authors' contributions}

AM participated in planning of the project, experimental data generation, data interpretation, manuscript writing, and provided some financial support. AS participated in planning of the project, experimental data generation, data interpretation, and editing of manuscript. ERG participated in experimental data generation. GB performed bioinformatics analysis. SP established various FISH/immunostaining procedures. KP conceived of the study and supervised the project, as well as participated in data interpretation, manuscript writing, and provided financial support. KP had final approval of the manuscript. All authors read and approved the final manuscript.

\section{Acknowledgements}

We thank Dr. Mitsuyoshi Nakao of Kumamoto University for kindly providing ATF7IP antibody. We thank Ryan Schmidt, Amy Pandya-Jones, and Vincent Pasque for experimental advice and reagent sharing. We are also grateful to Caroline Beard and Konrad Hochedlinger for help with the Hprt targeting strategy, and the UCLA transgenic core for blastocyst injection. KP is supported by the NIH (DP2OD001686 and P01 GM099134), CIRM (RN1-00564, RB3-05080, and RB4-06133), the Jonsson Comprehensive Cancer Center and the Eli and Edythe Broad Center of Regenerative Medicine and Stem Cell Research at UCLA. AM is supported by National Research Service Award AG039179, and SP by a CIRM training grant TG2-01169. KP and AM were supported for this work by funds from the Iris Cantor-UCLA Women's Health Center Executive Advisory Board. Funding bodies played no role in design, collection, analysis, or interpretation of data, nor in the writing of manuscript or decision to submit manuscript for publication.
Received: 2 March 2014 Accepted: 5 June 2014

Published: 26 June 2014

\section{References}

1. Wu H, Luo J, Yu H, Rattner A, Mo A, Wang Y, Smallwood PM, Erlanger B, Wheelan SJ, Nathans J: Cellular resolution maps of $X$ Chromosome inactivation: implications for neural development, function, and disease. Neuron 2014, 81:103-119.

2. Brockdorff N: Chromosome silencing mechanisms in X-chromosome inactivation: unknown unknowns. Development 2011, 138:5057-5065.

3. Pollex T, Heard E: Recent advances in X-chromosome inactivation research. Curr Opin Cell Biol 2012, 24:825-832.

4. Lee JT: Gracefully ageing at 50, X-chromosome inactivation becomes a paradigm for RNA and chromatin control. Nat Rev Mol Cell Biol 2011, 12:815-826.

5. Barakat TS, Gribnau J: $X$ chromosome inactivation in the cycle of life. Development 2012, 139:2085-2089.

6. Eggan K, Akutsu H, Hochedlinger K, Rideout W 3rd, Yanagimachi R, Jaenisch R: X-Chromosome inactivation in cloned mouse embryos. Science 2000, 290:1578-1581.

7. Maherali N, Sridharan R, Xie W, Utikal J, Eminli S, Arnold K, Stadtfeld M, Yachechko R, Tchieu J, Jaenisch R, Plath K, Hochedlinger K: Directly reprogrammed fibroblasts show global epigenetic remodeling and widespread tissue contribution. Cell Stem Cell 2007, 1:55-70.

8. Tada M, Takahama Y, Abe K, Nakatsuji N, Tada T: Nuclear reprogramming of somatic cells by in vitro hybridization with ES cells. Curr Biol 2001, 11:1553-1558.

9. Gendrel A-V, Apedaile A, Coker H, Termanis A, Zvetkova I, Godwin J, Tang YA, Huntley D, Montana G, Taylor S, Giannoulatou E, Heard E, Stancheva I, Brockdorff N: Smchd1-dependent and -independent pathways determine developmental dynamics of CpG island methylation on the inactive $X$ chromosome. Dev Cell 2012, 23:265-279.

10. Blewitt ME, Gendrel A-V, Pang Z, Sparrow DB, Whitelaw N, Craig JM, Apedaile A, Hilton DJ, Dunwoodie SL, Brockdorff N, Kay GF, Whitelaw E: SmcHD1, containing a structural-maintenance-of-chromosomes hinge domain, has a critical role in X inactivation. Nat Genet 2008, 40:663-669.

11. Casas-Delucchi CS, Brero A, Rahn H-P, Solovei I, Wutz A, Cremer T, Leonhardt $\mathrm{H}$, Cardoso MC: Histone acetylation controls the inactive X chromosome replication dynamics. Nat Commun 2011, 2:222.

12. Costanzi C, Pehrson JR: Histone macroH2A1 is concentrated in the inactive X chromosome of female mammals. Nature 1998, 393:599-601.

13. Heard E: Recent advances in X-chromosome inactivation. Curr Opin Cell Biol 2004, 16:247-255.

14. Hasegawa Y, Brockdorff N, Kawano S, Tsutui K, Tsutui K, Nakagawa S: The matrix protein hnRNP $\mathrm{U}$ is required for chromosomal localization of Xist RNA. Dev Cell 2010, 19:469-476.

15. Sado T, Okano M, Li E, Sasaki H: De novo DNA methylation is dispensable for the initiation and propagation of $\mathrm{X}$ chromosome inactivation. Development 2004, 131:975-982.

16. Kalantry S, Magnuson T: The Polycomb group protein EED is dispensable for the initiation of random X-chromosome inactivation. PLoS Genet 2006, 2:e66.

17. Leeb $M$, Wutz $A$ : Ring1B is crucial for the regulation of developmental control genes and PRC1 proteins but not $\mathrm{X}$ inactivation in embryonic cells. J Cell Biol 2007, 178:219-229.

18. Ohhata T, Tachibana M, Tada M, Tada T, Sasaki H, Shinkai Y, Sado T: X-inactivation is stably maintained in mouse embryos deficient for histone methyl transferase G9a. Genesis N Y N 2000 2004, 40:151-156.

19. Wutz $A$, Jaenisch $R: A$ shift from reversible to irreversible $X$ inactivation is triggered during ES cell differentiation. Mol Cell 2000, 5:695-705.

20. Csankovszki G, Nagy A, Jaenisch R: Synergism of Xist RNA, DNA methylation, and histone hypoacetylation in maintaining $X$ chromosome inactivation. J Cell Biol 2001, 153:773-784.

21. Hernández-Muñoz I, Lund AH, van der Stoop P, Boutsma E, Muijrers I, Verhoeven E, Nusinow DA, Panning B, Marahrens Y, van Lohuizen M: Stable $\mathrm{X}$ chromosome inactivation involves the PRC1 Polycomb complex and requires histone MACROH2A1 and the CULLIN3/SPOP ubiquitin E3 ligase. Proc Natl Acad Sci U S A 2005, 102:7635-7640.

22. Nusinow DA, Hernández-Muñoz I, Fazzio TG, Shah GM, Kraus WL, Panning B: Poly(ADP-ribose) Polymerase 1 Is Inhibited by a Histone H2A Variant, $\mathrm{MacroH} 2 \mathrm{~A}$, and Contributes to Silencing of the Inactive $\mathrm{X}$ Chromosome. J Biol Chem 2007, 282:12851-12859. 
23. Boggs BA, Cheung $P$, Heard E, Spector DL, Chinault AC, Allis CD: Differentially methylated forms of histone $\mathrm{H} 3$ show unique association patterns with inactive human X chromosomes. Nat Genet 2002, 30:73-76.

24. Heard E, Rougeulle C, Arnaud D, Avner P, Allis CD, Spector DL: Methylation of histone $\mathrm{H} 3$ at Lys-9 is an early mark on the $\mathrm{X}$ chromosome during $\mathrm{X}$ inactivation. Cell 2001, 107:727-738.

25. Mermoud JE, Popova B, Peters AHFM, Jenuwein T, Brockdorff N: Histone H3 lysine 9 methylation occurs rapidly at the onset of random $\mathrm{X}$ chromosome inactivation. Curr Biol 2002, 12:247-251.

26. Rougeulle C, Chaumeil J, Sarma K, Allis CD, Reinberg D, Avner P, Heard E: Differential Histone H3 Lys-9 and Lys-27 Methylation Profiles on the X Chromosome. Mol Cell Biol 2004, 24:5475-5484.

27. Escamilla-Del-Arenal M, da Rocha ST, Spruijt CG, Masui O, Renaud O, Smits AH, Margueron R, Vermeulen M, Heard E: Cdyl, a new partner of the inactive X Chromosome and potential reader of H3K27me3 and H3K9me2. Mol Cell Biol 2013, 33:5005-5020.

28. Cedar $\mathrm{H}$, Bergman $\mathrm{Y}$ : Linking DNA methylation and histone modification: patterns and paradigms. Nat Rev Genet 2009, 10:295-304.

29. Ichimura T, Watanabe S, Sakamoto Y, Aoto T, Fujita N, Nakao M: Transcriptional repression and heterochromatin formation by MBD1 and MCAF/AM family proteins. J Bio/ Chem 2005, 280:13928-13935.

30. Wang H, An W, Cao R, Xia L, Erdjument-Bromage H, Chatton B, Tempst P, Roeder RG, Zhang Y: mAM facilitates conversion by ESET of dimethyl to trimethyl lysine 9 of histone $\mathrm{H} 3$ to cause transcriptional repression. $\mathrm{Mol}$ Cell 2003, 12:475-487.

31. Fujita N, Watanabe S, Ichimura T, Ohkuma Y, Chiba T, Saya H, Nakao M: MCAF mediates MBD1-dependent transcriptional repression. Mol Cell Biol 2003, 23:2834-2843

32. Wade PA: Methyl CpG binding proteins: coupling chromatin architecture to gene regulation. Oncogene 2001, 20:3166-3173.

33. Beard C, Hochedlinger K, Plath K, Wutz A, Jaenisch R: Efficient method to generate single-copy transgenic mice by site-specific integration in embryonic stem cells. Genesis N Y N 2000 2006, 44:23-28.

34. Marahrens Y, Panning B, Dausman J, Strauss W, Jaenisch R: Xist-deficient mice are defective in dosage compensation but not spermatogenesis. Genes Dev 1997, 11:156-166.

35. Marahrens Y, Loring J, Jaenisch R: Role of the Xist Gene in X Chromosome choosing. Cell 1998, 92:657-664.

36. Yang $X$, Lay $F$, Han $H$, Jones PA: Targeting DNA methylation for epigenetic therapy. Trends Pharmacol Sci 2010, 31:536-546.

37. Hadjantonakis AK, Gertsenstein M, Ikawa M, Okabe M, Nagy A: Generating green fluorescent mice by germline transmission of green fluorescent ES cells. Mech Dev 1998, 76:79-90.

38. Itzkovitz $S$, van Oudenaarden A: Validating transcripts with probes and imaging technology. Nat Methods 2011, 8:S12-S19.

39. Plath K, Fang J, Mlynarczyk-Evans SK, Cao R, Worringer KA, Wang H, de la Cruz CC, Otte AP, Panning B, Zhang Y: Role of histone H3 lysine 27 methylation in X inactivation. Science 2003, 300:131-135.

40. Silva J, Mak W, Zvetkova I, Appanah R, Nesterova TB, Webster Z, Peters AHFM, Jenuwein T, Otte AP, Brockdorff N: Establishment of histone h3 methylation on the inactive $\mathrm{X}$ chromosome requires transient recruitment of Eed-Enx1 polycomb group complexes. Dev Cell 2003, 4:481-495.

41. Engreitz JM, Pandya-Jones A, McDonel P, Shishkin A, Sirokman K, Surka C, Kadri S, Xing J, Goren A, Lander ES, Plath K, Guttman M: The Xist IncRNA exploits three-dimensional genome architecture to spread across the $X$ Chromosome. Science 2013, 341:1237973.

42. Liu L, Ishihara K, Ichimura T, Fujita N, Hino S, Tomita S, Watanabe S, Saitoh N, Ito T, Nakao M: MCAF1/AM is involved in Sp1-mediated maintenance of cancer-associated telomerase activity. J Biol Chem 2009, 284:5165-5174.

43. Chang L-K, Chung J-Y, Hong Y-R, Ichimura T, Nakao M, Liu S-T: Activation of Sp1-mediated transcription by Rta of Epstein-Barr virus via an interaction with MCAF1. Nucleic Acids Res 2005, 33:6528-6539.

44. Masui $\mathrm{O}$, Heard E: RNA and protein actors in X-chromosome inactivation. Cold Spring Harb Symp Quant Biol 2006, 71:419-428.

45. Pullirsch D, Härtel R, Kishimoto $H$, Leeb $M$, Steiner $G$, Wutz A: The Trithorax group protein Ash2l and Saf-A are recruited to the inactive $X$ chromosome at the onset of stable X inactivation. Development 2010, 137:935-943.

46. De Graeve F, Bahr A, Chatton B, Kedinger C: A murine ATFa-associated factor with transcriptional repressing activity. Oncogene 2000, 19:1807-1819.
47. Li E: Chromatin modification and epigenetic reprogramming in mammalian development. Nat Rev Genet 2002, 3:662-673.

48. Dodge JE, Kang Y-K, Beppu H, Lei H, Li E: Histone H3-K9 methyltransferase ESET is essential for early development. Mol Cell Biol 2004, 24:2478-2486.

49. Zhao X, Ueba T, Christie BR, Barkho B, McConnell MJ, Nakashima K, Lein ES, Eadie BD, Willhoite AR, Muotri AR, Summers RG, Chun J, Lee K-F, Gage FH: Mice lacking methyl-CpG binding protein 1 have deficits in adult neurogenesis and hippocampal function. Proc Natl Acad Sci U S A 2003, 100:6777-6782

50. Waterfield M, Khan IS, Cortez JT, Fan U, Metzger T, Greer A, Fasano K, Martinez-Llordella M, Pollack JL, Erle DJ, Su M, Anderson MS: The transcriptional regulator Aire coopts the repressive ATF7ip-MBD1 complex for the induction of immunotolerance. Nat Immunol 2014, 15:258-265.

51. Chadwick BP, Willard HF: Multiple spatially distinct types of facultative heterochromatin on the human inactive $\mathrm{X}$ chromosome. Proc Natl Acad Sci U S A 2004, 101:17450-17455.

52. Nozawa R-S, Nagao K, Igami K-T, Shibata S, Shirai N, Nozaki N, Sado T, Kimura $\mathrm{H}$, Obuse $\mathrm{C}$ : Human inactive $\mathrm{X}$ chromosome is compacted through a PRC2-independent SMCHD1-HBiX1 pathway. Nat Struct Mol Biol 2013, 20:566-573.

53. Lehnertz B, Ueda Y, Derijck AAHA, Braunschweig U, Perez-Burgos L, Kubicek S, Chen T, Li E, Jenuwein T, Peters AHFM: Suv39h-mediated histone H3 lysine 9 methylation directs DNA methylation to major satellite repeats at pericentric heterochromatin. Curr Biol 2003, 13:1192-1200.

54. Csankovszki G, Panning B, Bates B, Pehrson JR, Jaenisch R: Conditional deletion of Xist disrupts histone macroH2A localization but not maintenance of X inactivation. Nat Genet 1999, 22:323-324.

doi:10.1186/1756-8935-7-12

Cite this article as: Minkovsky et al:: The Mbd1-Atf7ip-Setdb1 pathway contributes to the maintenance of $\mathrm{X}$ chromosome inactivation. Epigenetics \& Chromatin 2014 7:12.

\section{Submit your next manuscript to BioMed Central and take full advantage of:}

- Convenient online submission

- Thorough peer review

- No space constraints or color figure charges

- Immediate publication on acceptance

- Inclusion in PubMed, CAS, Scopus and Google Scholar

- Research which is freely available for redistribution 